\title{
Plumbagin inhibits tumor angiogenesis of gastric carcinoma in mice by modulating nuclear factor-kappa B pathway
}

\author{
Chengqian Yang ${ }^{1}$, Xingbo Feng ${ }^{2}$, Zengxian $\mathrm{Li}^{1}$, Qingsi $\mathrm{He}^{3}$ \\ ${ }^{1}$ Department of General Surgery, the Second Hospital of Shandong University, Jinan 250033, China; ${ }^{2}$ Department of Gastrointestinal Surgery, the \\ Central Hospital of Zaozhuang Mining Group, Zaozhuang 277101, China; ${ }^{3}$ Department of Gastrointestinal Surgery, Qilu Hospital of Shandong \\ University, Jinan 250012, China \\ Contributions: (I) Conception and design: C Yang, Q He; (II) Administrative support: None; (III) Provision of study materials or patients: C Yang, \\ X Feng; (IV) Collection and assembly of data: All authors; (V) Data analysis and interpretation: X Feng, Z Li; (VI) Manuscript writing: All authors; \\ (VII) Final approval of manuscript: All authors. \\ Correspondence to: Qingsi He. Department of Gastrointestinal Surgery, Qilu Hospital of Shandong University, No.107, Cultural West Road, Lixia \\ District, Jinan 250012, China. Email: qsh852147@126.com.
}

\begin{abstract}
Background: We aimed to determine the mechanism of plumbagin on tumor growth of gastric carcinoma. Methods: Sixty BALB/c mice were treated with $200 \mu \mathrm{L}$ SGC-7901 cells to establish gastric carcinoma xenograft and randomly divided into four groups: model group, low dose of plumbagin group (2 mg/kg), medium dose of plumbagin group $(4 \mathrm{mg} / \mathrm{kg})$ and high dose of plumbagin group $(6 \mathrm{mg} / \mathrm{kg})$. The tumor volume and weight were measured every week, and the ratio of anti-tumor was analyzed. The contents of vascular endothelial growth factor (VEGF), VEGF receptor-2 (VEGFR2) in serum and tumor tissues were tested by enzyme linked immunosorbent assay (ELISA), immunohistochemistry and Western blot, respectively. The microvessel density (MVD) in tumor tissues was evaluated by immunohistochemistry and

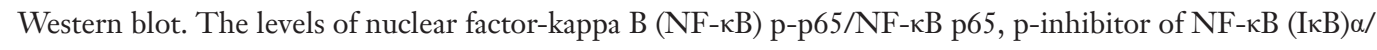
$\mathrm{IKB} \alpha, \mathrm{p}-\mathrm{I} \kappa \mathrm{B}$ kinase (IKK)/IKK in tumor tissues were also analyzed by Western blot.

Results: Compared with model group, plumbagin treatment significantly suppressed the growth of tumor $(\mathrm{P}<0.05)$. The inhibition rate was $50.32 \%$ in high dose group $(6 \mathrm{mg} / \mathrm{kg})$. Furthermore, we found that the expressions of VEGF, VEGRF2 and MVD were obviously decreased in plumbagin treatment groups when compared to model group $(\mathrm{P}<0.05)$. Importantly, plumbagin treatment could down-regulate the levels of

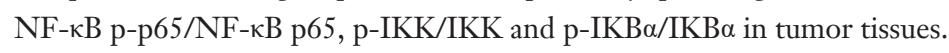

Conclusions: Our study indicated that plumbagin might be an effective drug in inhibiting the tumor angiogenesis of gastric cancer and the mechanism of anti-tumor may be associated with NF- $\mathrm{BB}$ pathway.

Keywords: Plumbagin; tumor angiogenesis; gastric cancer; nuclear factor-kappa B (NF-кB)

Submitted Jul 25, 2019. Accepted for publication Nov 11, 2019.

doi: $10.21037 /$ tcr.2019.12.03

View this article at: http://dx.doi.org/10.21037/tcr.2019.12.03

\section{Introduction}

Gastric cancer, the second incidence in the cancers, is one of the most common malignancies worldwide, with high rate of incidence, metastasis and mortality and low rate of early diagnosis (1). Despite many approaches have been used to treat the disease, including surgical resection, neoadjuvant chemotherapy, radiotherapy, molecular-targeted therapies and immunotherapy, the overall 5 -year survival rate of gastric cancer patients is less than $20 \%(1,2)$. Tumor angiogenesis is an important step in tumor progress, which supports the cancer growth and metastasis (3). Inhibiting angiogenesis is a promising strategy for the anticancer drugs, but there were few available drugs for patients with gastric cancer.

As a natural constituent, plumbagin (5-hydroxy-2- 
methyl-1,4-napthoquinone) is isolated from the roots of medicinal plant, Plumbago zeylanica L. (4). Many reports have explored that plumbagin had various abilities, including antioxidation, anti-inflammatory, radiosensitization (5). Furthermore, numerous researches have shown that plumbagin could inhibit the progression of many cancers, such as esophageal squamous cell carcinoma (ESCC) (4), ovarian cancer (6), colorectal carcinoma and prostate cancer (7). Plumbagin also contributed to other diseases treatment, such as cerebral ischemia (8), diabetic nephropathy (9). However, there is little research to study the effect of plumbagin on gastric cancer.

Many studies have shown that the aggressiveness of gastric cancer is associated with the activity of nuclear factor-kappa $\mathrm{B}(\mathrm{NF}-\mathrm{\kappa B})$ transcription factor. These studies found that NF- $\kappa \mathrm{B}$ pathway was activated and promoted gastric cancer progression (10-12). The NF-кB pathway regulated inflammation, cell survival, apoptosis and proliferation in gastric cancer, which was the key factor for precancerous and malignant cells to escape apoptosis (12). Angiogenesis, the growth of new blood vessels from pre-existing one, plays a pivotal role in tumor growth and metastasis. It is reported that plumbagin induced vascular endothelial growth factor receptor-2 (VEGFR2) expression to suppress the migration and invasion in tumor derikced endothelial cells (7), ovarian cancer (6). However, little information is reported on the effects of plumbagin on gastric cancer angiogenesis.

In this study, we hypothesized that plumbagin could inhibit gastric cancer angiogenesis by mediating NF- $\mathrm{NB}$ pathway. We used nude mice to explore the hypothesis.

\section{Methods}

\section{Cells and animals}

The SGC-7901 cells were obtained from American Type Culture Collection (ATCC) and cultured in Dulbecco's Modified Eagle's Medium (DMEM) medium (Gibco,Rockville, MD, USA) with 10\% fetal bovine serum (FBS) (Sigma-Aldrich, St. Louis, MO, USA) at $37{ }^{\circ} \mathrm{C}, 5 \%$ $\mathrm{CO} 2$. When the cells were full of monolayer, the cells were digested with $0.25 \%$ trypsin (Solarbio, Beijing, China) and centrifuged for $1,000 \mathrm{r} / \mathrm{min}, 5 \mathrm{~min}$. The concentration of cells was $1 \times 10^{7}$ cells $/ \mathrm{mL}$.

Female BALB/c (nu/nu) mice (4-6 weeks, 2630 g, Changzhou Cavens Laboratory Animal Co., Ltd., $\operatorname{SCXK}(\mathrm{Su}) 20160010)$ were raised in standard environment,
$20-26{ }^{\circ} \mathrm{C}$, humidity $40-60 \%, 12 \mathrm{~h}$ light/dark cycle with free access to food and water. All animal experiments were approved by the Institutional Animal Care and Use Committee of the Second Hospital of Shandong University (No. SHSU-20180721003).

\section{Animal model and groups}

Sixty mice were injected subcutaneously with $200 \mu \mathrm{L}$ SGC-7901 cells to the right forelimb. After that, the mice were put back into cage and fed again until $5 \mathrm{~mm}$ nodules appeared subcutaneously.

The mice were randomly divided into model group (M), low dose of plumbagin group (P-L, $2 \mathrm{mg} / \mathrm{kg}$ ), medium dose of plumbagin group (P-M, $4 \mathrm{mg} / \mathrm{kg}$ ) and high dose of plumbagin group (P-H, $6 \mathrm{mg} / \mathrm{kg}), 15$ mice in each group. All mice were given intraperitoneal injection once a day for 6 weeks. In model group, the mice were given the same volume of saline.

\section{Tumor size and tumor weight}

The animal health and behavior were monitored every week. The volume of tumor was calculated every week and the formula was following: $\mathrm{V}\left(\mathrm{mm}^{3}\right)=0.5 \times$ length $\times$ width $^{2}$. The weight of mice in each group was weighed and the eyeballs were removed to collect blood. All mice were anesthetized by intraperitoneal injection of $10 \%$ chloral hydrate $(3 \mathrm{mg} / \mathrm{kg})$ at the end of the 6th week. And there was no animal exhibited signs of peritonitis. The mice were sacrificed by cervical dislocation and no heartbeat for 5 min meant death. Tumors were dissected for immunohischemical staining and western blot.

\section{Enzyme linked immunosorbent assay (ELISA)}

Serum was collected by centrifugation, then the levels of VEGF, VEGF receptor-2 (VEGFR2) in serum were determined by the ELISA kits (Mibio, Shanghai, China).

\section{Immunobistochemical staining}

Tumor sections $(4 \mu \mathrm{m})$ were dewaxed and hydrated as conventional administration. After that, the sections were washed with phosphate-buffered saline (PBS, Baso, Zhuhai, China) three times, 5 min each time. Antigen was renovated at $95^{\circ} \mathrm{C}, 60 \mathrm{~min}$ and cooled to $25^{\circ} \mathrm{C}, 3 \mathrm{~min}$, three times. Primary antibodies CD31 (1:100, Cell signaling technology, 
Danvers, MA, USA), VEGF (1:400, VC001, R\&D systems, Shanghai, China), VEGFR2 (1:400, AF644, R\&D systems, Shanghai, China) were incubated at $4{ }^{\circ} \mathrm{C}, 24 \mathrm{~h}$. Then, the sections were incubated at $37{ }^{\circ} \mathrm{C}$ for $30 \mathrm{~min}$ before washed with phosphate buffered saline (PBST). Washing $5 \mathrm{~min}$, the sections were incubated with HRP polymer (1:400, R\&D systems, Shanghai, China) at $37^{\circ} \mathrm{C}, 30 \mathrm{~min}$. Washing 5 min with PBST, the slices were cultured with DAB Horseradish Peroxidase Color Development Kit (Beyotime Biotechnology, Shanghai, China) for 3-15 min. Positive expression areas were observed under microscope (DMi8, Feica Microsystems, Germany).

\section{Western blot}

The proteins of tumor tissues were performed by building and construction authority (BCA) kit (Pierce Company). Samples from each group were isolated by SDS-PAGE electrophoresis (Mini-Protean-3, bio-Rad, Hercules, CA, USA) and then transferred to PVDF membrane (Millipore, Massachusetts, USA). Skim milk of $5 \%$ was used for sealing in $1 \mathrm{~h}$. After washing with PBS, membranes were incubated with antibodies: anti-VEGF antibody $(1: 1,500$, AF-493-NA, R\&D Systems), anti-VEGFR2 antibody (1:1,000, ab11939, Abcam), anti-CD31 antibody (1:800; AF3628, R\&D Systems), anti-NF-kB p-p65 antibody (1:1,000, ab86299, Abcam), anti-NF-kB p65 antibody (1:1,000, ab19870, Abcam), anti-p-inhibitor of NF- $\mathrm{B}$ (IкB) $\alpha$ antibody (1:2,000, ab19870, Abcam), anti-inhibitor

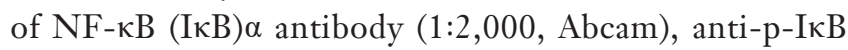
kinase (IKK) antibody (1:2,000, ab17943, Abcam), anti IKK antibody $(1: 2,000$, ab17943, Abcam $)$ and anti- $\beta$ actin $\left(1: 1,000\right.$, ab179467, Abcam) overnight at $4{ }^{\circ} \mathrm{C}$. The membranes were washed with PBS and incubated with IgG-HRP secondary antibody (1:800, ab205719, Abcam) at room temperature for $1 \mathrm{~h}$. Signals detection was performed using ECL luminescent substrate (Thermo Fisher Scientific, Inc., Shanghai, China). Relative expression levels of each protein were normalized to $\beta$-actin.

\section{Statistical analysis}

SPSS20.0 software was used to perform all data and data was expressed as $(\bar{x} \pm \mathrm{SD})$. One-way analysis of variance (ANOVA) was used to compare among groups. The Dunnett test was used in subsequent analysis. $\mathrm{P}<0.05$ was statistically significant.

\section{Results}

\section{Plumbagin suppressed the growth of tumor}

During the experiment, the volume of tumors in each group increased in varying degrees. As shown in Figure $1 \mathrm{~A}$, the animal weight was decreased rapidly in model group, while animal weight was increased after plumbagin treatment. The tumor volume increased rapidly in model group (Figure 1B). After treatment with plumbagin, the growth rate of tumors was relatively slow. Especially, the tumor growth trend was obviously suppressed in $\mathrm{P}-\mathrm{H}$ group $(\mathrm{P}<0.05$, Figure $1 B)$. The maximum diameter of tumors was $1.48 \mathrm{~cm}$ in model group, $1.32 \mathrm{~cm}$ in $\mathrm{P}-\mathrm{L}$ group, $1.21 \mathrm{~cm}$ in $\mathrm{P}-\mathrm{M}$ group, and $0.87 \mathrm{~cm}$ in $\mathrm{P}-\mathrm{H}$ group (Figure $1 \mathrm{C}$ ). Furthermore, the tumor weight in different dose of plumbagin groups was significantly lower than that in model group $(\mathrm{P}<0.05$, Figure $1 C)$. The inhibition rates in low, medium and high doses of plumbagin group were $31.85 \%, 40.76 \%$ and $50.32 \%$, respectively (Table 1 ).

\section{Plumbagin decreased the expression of VEGF and VEGFR2 in serum and tumor}

The results of VEGF and VEGFR2 expression in serum and tumor were shown in Figure 2. Compared with model group, the levels of VEGF and VEGFR2 in serum were significantly decreased after different doses of plumbagin treatment (Figure $2 A, \mathrm{P}<0.05$ ). The treatment effect was developed in dose manner. Consistent with the expression in serum, the expression of VEGF and VEGFR2 in tumor was also markedly declined in different plumbagin treatment groups when compared to model group (Figure $2 B, \mathrm{P}<0.05$ ).

\section{Plumbagin reduced the microvessel density (MVD) in tumor}

The expression of CD31 in vascular endothelial cells represents the expression of MVD, so we observed the CD31 expression in tumor tissues by immunohistochemistry (Figure $3 A$ ) and Western blot (Figure 3B). The positive expression of CD31 was the most obvious in model group, and the trend reversed after different doses of plumbagin administration $(\mathrm{P}<0.05)$. 

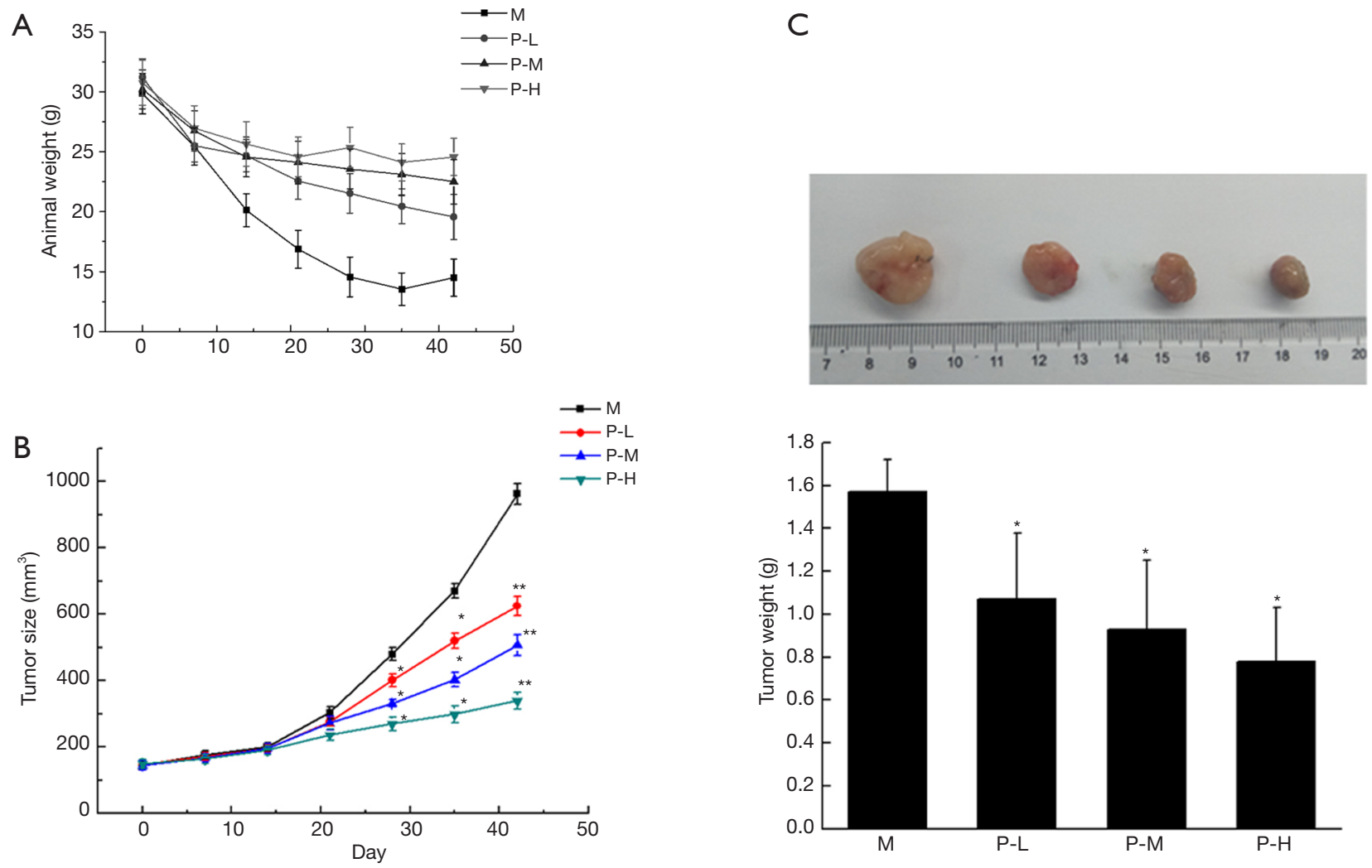

Figure 1 The effect of plumbagin on the growth of gastric cancer xenografts in nude mice (n=15). (A) Animal weight; (B) tumor size; (C) tumor weight. * $\mathrm{P}<0.05$; **, $\mathrm{P}<0.01$ compared with model group. $\mathrm{M}$, model group; $\mathrm{P}-\mathrm{L}$, plumbagin low dose group; $\mathrm{P}-\mathrm{M}$, plumbagin medium dose group; $\mathrm{P}-\mathrm{H}$, plumbagin high dose group.

Table 1 Tumor weight and inhibition rate of tumor in groups $(\mathrm{n}=15$, $\left.\bar{x}_{ \pm} \mathrm{SD}\right)$

\begin{tabular}{lcc}
\hline Group $(\mathrm{n}=15)$ & Plumbagin $(\mathrm{mg} / \mathrm{kg})$ & Inhibition rate $(\%)$ \\
\hline Model & - & 0 \\
Plumbagin low & 2 & 31.85 \\
Plumbagin medium & 4 & 40.76 \\
Plumbagin high & 6 & 50.32 \\
\hline
\end{tabular}

*, $\mathrm{P}<0.05$, compared with model group.

\section{Plumbagin reduced the expression of VEGF and VEGFR2 in tumor}

In order to confirm that plumbagin treatment decreased the expression of VEGF and VEGFR2 in tumors, we analyzed the expression of above proteins by immunohistochemistry (Figure 4). Similar with the results of Figure 2, the positive expression of VEGF and VEGFR2 in model group was the most obvious when compared to other groups. However, the expression of VEGF and VEGFR2 was decreased gradually with increasing dosage of plumbagin treatment.

\section{Plumbagin regulated the expression of $\mathrm{NF}-\kappa \mathrm{B}$ patbway in} tumor

The expression of NF- $\kappa \mathrm{B}$ p-p65/ NF- $\kappa \mathrm{B}-\mathrm{p} 65$, p-IKK/ IKK and IKB $\alpha / \mathrm{IKB} \alpha$ in tumor was analyzed by Western blot (Figure 5). Compared with model group, the levels of NF-kB p-p65/NF- $\mathrm{BB}-\mathrm{p} 65, \mathrm{p}-\mathrm{IKK} / \mathrm{IKK}$ and p-IKB $\alpha /$ $\mathrm{IKB} \alpha$ were evidently decreased in medium and high dose of plumbagin groups $(\mathrm{P}<0.05)$. These proteins were expressed in a dose-dependent manner. 

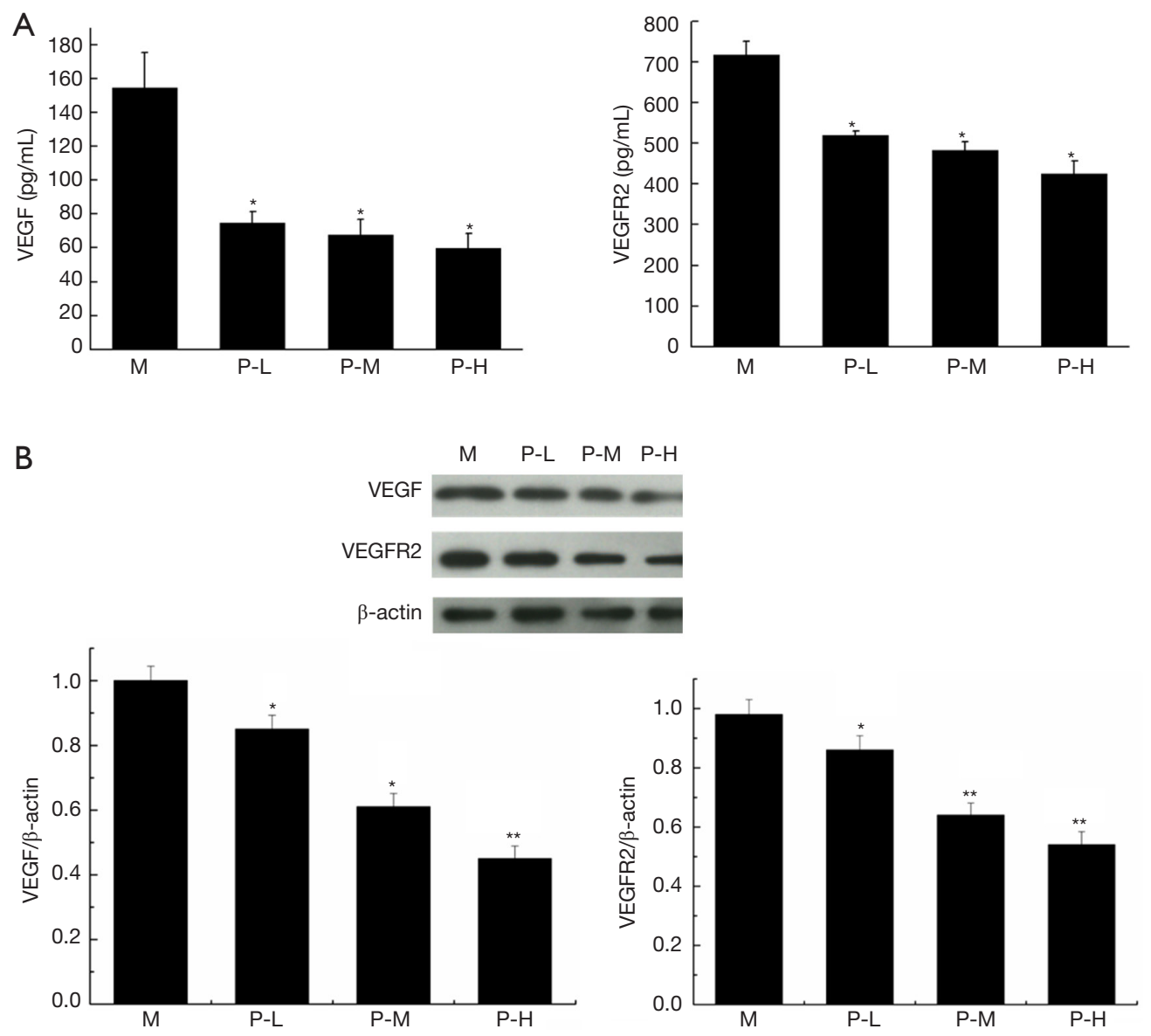

Figure 2 The effect of plumbagin on the levels of VEGF and VEGFR2 in serum and tumor tissues (n=15). (A) The levels in serum analyzed by ELISA; (B) the expression in tumor tissues analyzed by Western blot. *, $\mathrm{P}<0.05$; **, $\mathrm{P}<0.01$ compared with model group. M, model group; $\mathrm{P}-\mathrm{L}$, plumbagin low dose group; P-M, plumbagin medium dose group; P-H, plumbagin high dose group; VEGF, vascular endothelial growth factor; VEGFR2, VEGF receptor-2; ELISA, enzyme linked immunosorbent assay.

\section{Discussion}

In our research, we found plumbagin could suppress the gastric cancer growth via inhibiting angiogenesis and NF$\kappa \mathrm{B}$ pathway. These results were consistent with previous studies, which have showed that plumbagin could inhibit tumorigenesis and angiogenesis in different cancer types, including esophageal cancer, ovarian cancer $(4,6,7,13)$. As known, endothelial cell was the major components of blood vessels, which played an important role in angiogenesis of tumor (14). VEGF and its receptor VEGFR2 were important factors for angiogenesis, and they were extensively studied in many researches $(6,15)$. In these studies, scholars found that the expression of VEGF and its receptor VEGFR2 were overexpressed in many tumors, such as ovarian cancer (6), breast cancer (15), hepatocellular carcinoma (6). The two factors take part in tumor growth, and inhibition the expression of them was contributed to cancer treatment. In this study, we identified plumbagin inhibited angiogenesis of gastric cancer via preventing the expression of VEGF, VEGFR2 in serum and tumor tissue, which suggested plumbagin played a protective role in tube formation of gastric cancer.

It has been widely known, $\mathrm{NF}-\kappa \mathrm{B}$ signaling contributes to cancer process by promoting tumor microenvironment (14). The NF- $\kappa \mathrm{B}$ pathway is involved in the regulation of multiple cellular processes, including cell proliferation, migration and survival. This pathway is also a significant 

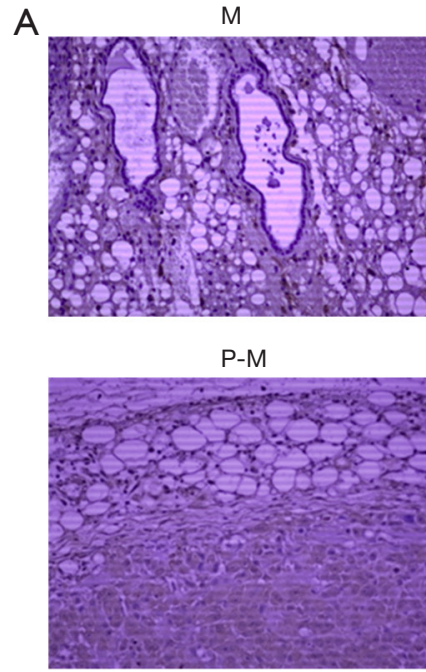

B

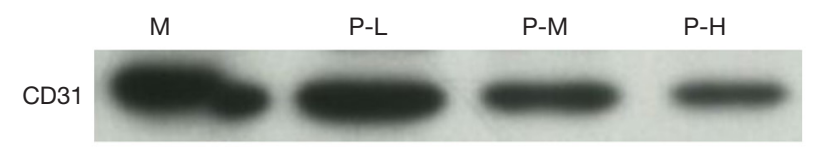

$\beta$-actin

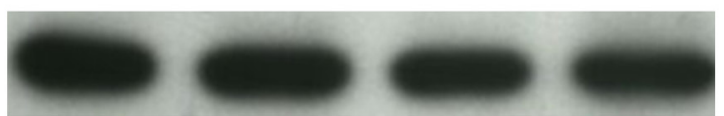

P-L

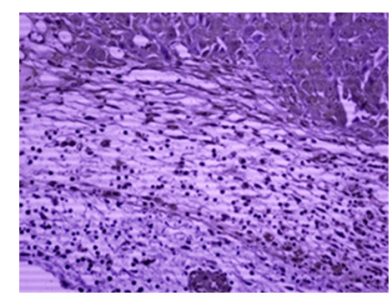

P-H

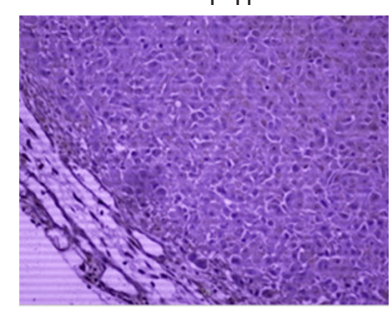

P-M P-H

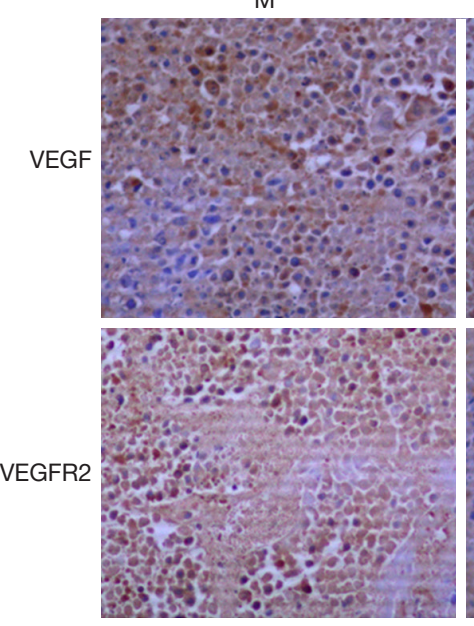

P-L
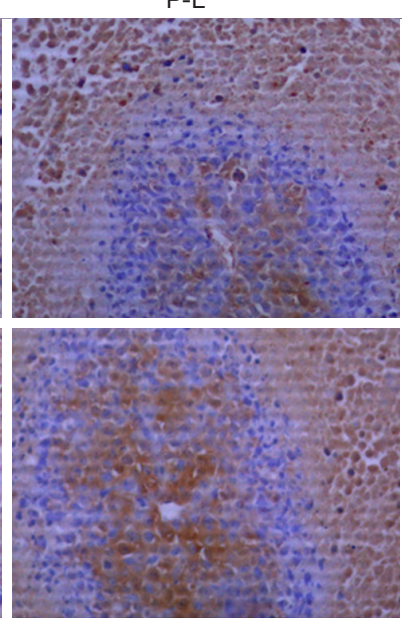
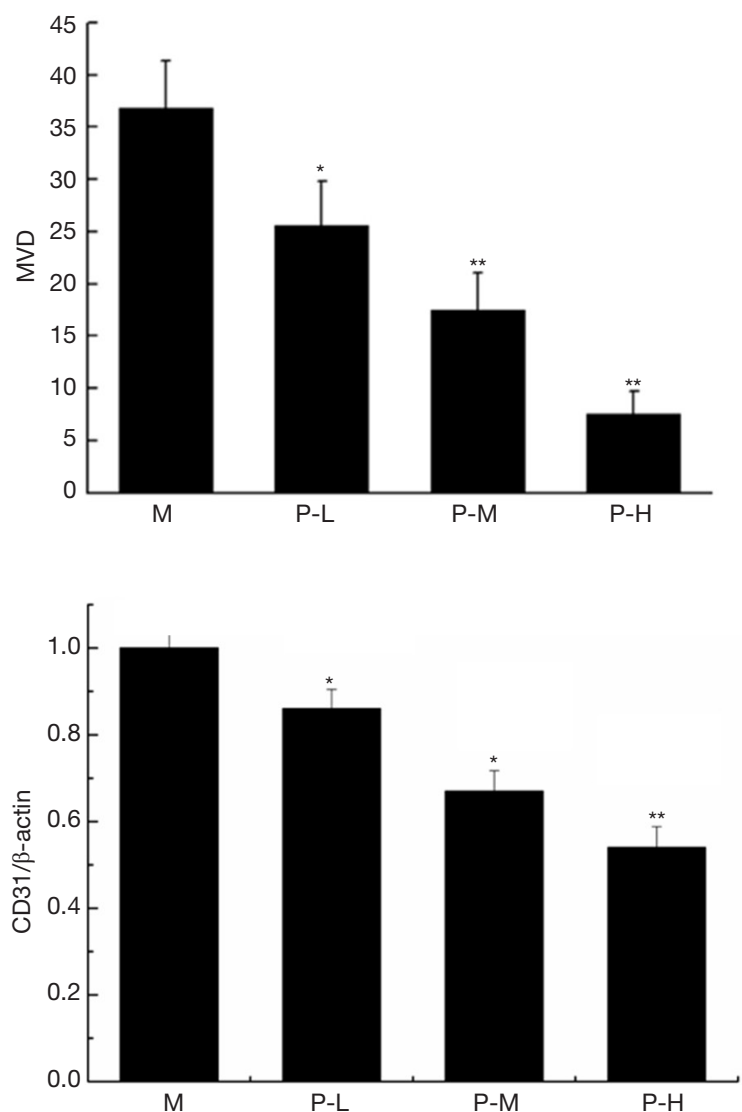

Figure 3 The effect of plumbagin on MVD in transplanted tumors ( $\mathrm{n}=5)$. (A) tumor angiogenesis was assessed by IHC, magnification 100x. (B) the expression of CD31 was measured by Western blot. * $\mathrm{P}<0.05$; **, $\mathrm{P}<0.01$ compared with model group. M, model group; $\mathrm{P}-\mathrm{L}$, plumbagin low dose group; P-M, plumbagin medium dose group; $\mathrm{P}-\mathrm{H}$, plumbagin high dose group; MVD, microvessel density.

Figure 4 The effect of plumbagin on VEGF and VEGFR2 of transplanted tumors in nude mice (n=5), IHC staining, magnification 100×. M, model group; P-L, plumbagin low dose group; P-M, plumbagin medium dose group; P-H, plumbagin high dose group; VEGF, vascular endothelial growth factor; VEGFR2, VEGF receptor-2. 
A
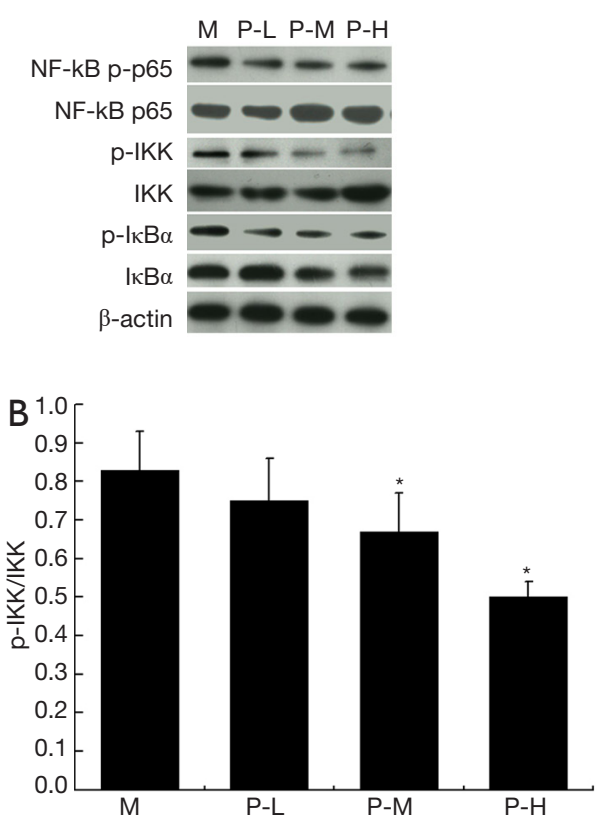
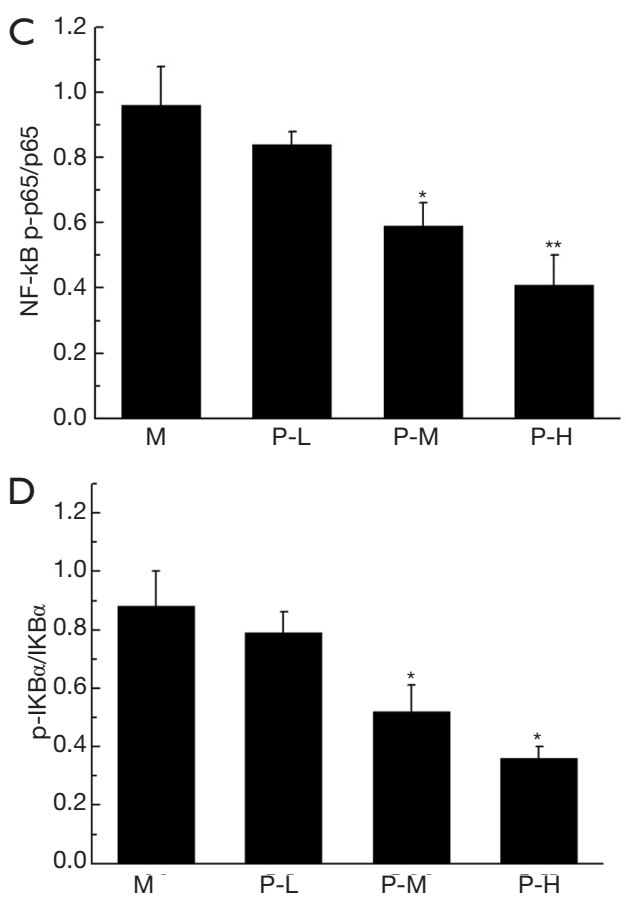

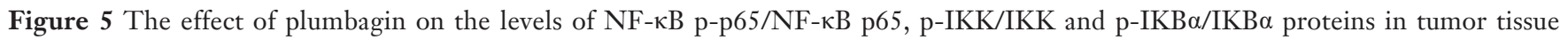
$(n=5)$. (A) the expression of proteins was analyzed by Western blot; $(B, C, D)$ an anti- $\beta$-actin antibody was used to confirm equivalent protein loading. *, $\mathrm{P}<0.05$; ** $\mathrm{P}<0.01$ compared with model group. $\mathrm{M}$, model group; $\mathrm{P}-\mathrm{L}$, plumbagin low dose group; $\mathrm{P}-\mathrm{M}$, plumbagin medium dose

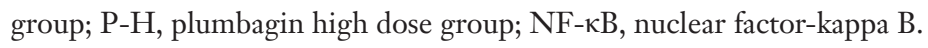

contributor to blood vessel formation. It found that plumbagin promoted the expression of PD-L1 in gastric cancer cells through activating NF- $\kappa \mathrm{B}$ pathway (16).

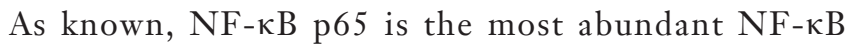
heterodimer, which is sequestered in the cytoplasm with I $\mathrm{B}$ proteins when it is in inactive state. The IKK complex is associated with the activation of NF- $\kappa \mathrm{B}(17)$. The phosphorylation of $\mathrm{I} \kappa \mathrm{B} \alpha$ participates in the activation of $\mathrm{NF}-\kappa \mathrm{B}$, leading to tumor degradation. A report showed that plumbagin suppressed the invasion of breast cancer cells through inhibition of IKB $\alpha$ mediated NF- $\kappa \mathrm{B}$ activation (18). In our study, we also found that plumbagin could suppress the phosphorylation of NF- $\kappa \mathrm{B}$ p 65 , IKK and I $\mathrm{BB} \alpha$ in gastric cancer. These results were consistent with previous studies, and suggested that plumbagin suppressed the growth of gastric cancer might be by mediating the NF- $\mathrm{\kappa B}$ pathway.

Recent reviewers found that VEGF could induce the activation of Akt, ERK1/2, NF- $\mathrm{kB}$ and AMPK pathways in H460 cells (19). These reviewers proposed VEGF binds to its receptor (VEGFR) to further induce the activation of the downstream signals in tumor endothelial cells, including the extracellular regulated protein kinases (ERK), NF$\kappa \mathrm{B}$ and threonine protein kinase $(\mathrm{Akt}) /$ mammalian target of rapamycin (mTOR) pathways. Constitutively, NF$\kappa \mathrm{B}$ activation was associated with anti-apoptotic and proangiogenesis related genes expression, such as cyclin D1, VEGF $(20,21)$. Our results showed NF-кB signaling was activated and VEGF was overexpressed in gastric cancer, but whether VEGF promoted phosphorylation of NF- $\mathrm{\kappa B}$ $\mathrm{p} 65$, IKK and I $\kappa \mathrm{B} \alpha$ need to modify in the future study. In addition, whether plumbagin has side effects for treatment gastric cancer? That is also need to find.

\section{Conclusions}

In this study, we found plumbagin could significantly delay the growth of gastric tumors via reducing microvessel density and decreasing the expression of NF- $\kappa \mathrm{B}$ pathway in tumors. These results suggest that plumbagin may be a potential drug for treatment of gastric cancer. 


\section{Acknowledgments}

Funding: The study was supported by Youth Fund of the Second Hospital of Shandong University (Y2013010056).

\section{Footnote}

Conflicts of Interest: The authors have completed the ICMJE uniform disclosure form (available at http://dx.doi. org/10.21037/tcr.2019.12.03). The authors have no conflicts of interest to declare.

Ethical Statement: The authors are accountable for all aspects of the work in ensuring that questions related to the accuracy or integrity of any part of the work are appropriately investigated and resolved. All animal experiments were approved by the Institutional Animal Care and Use Committee of the Second Hospital of Shandong University (No. SHSU-20180721003).

Open Access Statement: This is an Open Access article distributed in accordance with the Creative Commons Attribution-NonCommercial-NoDerivs 4.0 International License (CC BY-NC-ND 4.0), which permits the noncommercial replication and distribution of the article with the strict proviso that no changes or edits are made and the original work is properly cited (including links to both the formal publication through the relevant DOI and the license). See: https://creativecommons.org/licenses/by-nc$\mathrm{nd} / 4.0 /$.

\section{References}

1. Song Z, Wu Y, Yang J, et al. Progress in the treatment of advanced gastric cancer. Tumour Biol 2017;39:1010428317714626.

2. Yan W, Chen S, Zhao Y, et al. Fisetin inhibits the proliferation of gastric cancer cells and induces apoptosis through suppression of ERK $1 / 2$ activation. Oncol Lett 2018;15:8442-6.

3. Wang C, Li J, Ye S, et al. Oestrogen Inhibits VEGF Expression And Angiogenesis In Triple-Negative Breast Cancer By Activating GPER-1. J Cancer 2018;9:3802-11.

4. Cao YY, Yu J, Liu TT, et al. Plumbagin inhibits the proliferation and survival of esophageal cancer cells by blocking STAT3-PLK1-AKT signaling. Cell Death Dis 2018;9:17.
5. Wang KH, Li BZ. Plumbagin protects against hydrogen peroxide-induced neurotoxicity by modulating NF- $\kappa \mathrm{B}$ and Nrf-2. Arch Med Sci 2018;14:1112-8.

6. Sinha S, Pal K, Elkhanany A, et al. Plumbagin inhibits tumorigenesis and angiogenesis of ovarian cancer cells in vivo. Int J Cancer 2013;132:1201-12.

7. Lai L, Liu J, Zhai D, et al. Plumbagin inhibits tumour angiogenesis and tumour growth through the Ras signalling pathway following activation of the VEGF receptor-2. Br J Pharmacol 2012;165:1084-96.

8. Chen XJ, Zhang JG, Wu L. Plumbagin inhibits neuronal apoptosis, intimal hyperplasia and also suppresses TNF- $\alpha$ / $\mathrm{NF}-\kappa \mathrm{B}$ pathway induced inflammation and matrix metalloproteinase-2/9 expression in rat cerebral ischemia. Saudi J Biol Sci 2018;25:1033-9.

9. Yong R, Chen XM, Shen S, et al. Plumbagin ameliorates diabetic nephropathy via interruption of pathways that include NOX4 signalling. PLoS One 2013;8:e73428.

10. Wu L, Zhang X, Zhang B, et al. Exosomes derived from gastric cancer cells activate $\mathrm{NF}-\kappa \mathrm{B}$ pathway in macrophages to promote cancer progression. Tumour Biol 2016;37:12169-80.

11. Zhi Q, Chen H, Liu F, et al. Podocalyxin-like protein promotes gastric cancer progression through interacting with RUN and FYVE domain containing 1 protein. Cancer Sci 2019;110:118-34.

12. Han MY, Nie JW, Li YY, et al. Downregulation of NGAL is Required for the Inhibition of Proliferation and the Promotion of Apoptosis of Human Gastric Cancer MGC-803 Cells. Cell Physiol Biochem 2018;50:694-705.

13. Martins GR, Gelaleti GB, Moschetta MG, et al. Proinflammatory and Anti-Inflammatory

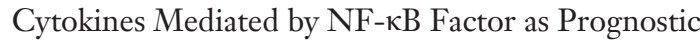
Markers in Mammary Tumors. Mediators Inflamm 2016;2016:9512743.

14. Liu G, Kuang S, Wu S, et al. A novel polysaccharide from Sargassum integerrimum induces apoptosis in A549 cells and prevents angiogensis in vitro and in vivo. Sci Rep 2016;6:26722.

15. Ciccone V, Terzuoli E, Donnini S, et al. Stemness marker ALDH1A1 promotes tumor angiogenesis via retinoic acid/ HIF-1 $\alpha$ /VEGF signalling in MCF-7 breast cancer cells. J Exp Clin Cancer Res 2018;37:311.

16. Wu C, Lv C, Chen F, et al. The function of miR-199a-5p/ Klotho regulating TLR4/NF- $\kappa \mathrm{B}$ p65/NGAL pathways 
in rat mesangial cells cultured with high glucose and the mechanism. Mol Cell Endocrinol 2015;417:84-93.

17. Durand JK, Baldwin AS. Targeting IKK and NF- $\mathrm{KB}$ for Therapy. Adv Protein Chem Struct Biol 2017;107:77-115.

18. Kawiak A, Domachowska A. Plumbagin Suppresses the Invasion of HER2-Overexpressing Breast Cancer Cells through Inhibition of IKK $\alpha-$ Mediated NF- $\kappa \mathrm{B}$ Activation. PLoS One 2016;11:e0164064.

19. Wang JC, Chen SY, Wang M, et al. Nickel-induced VEGF expression via regulation of Akt, ERK1/2, NFкB, and AMPK pathways in H460 cells. Environ Toxicol

Cite this article as: Yang C, Feng X, Li Z, He Q. Plumbagin inhibits tumor angiogenesis of gastric carcinoma in mice by modulating nuclear factor-kappa B pathway. Transl Cancer Res 2020;9(2):556-564. doi: 10.21037/tcr.2019.12.03
2019;34:652-8.

20. Hsu FT, Chiang IT, Kuo YC, et al. Amentoflavone Effectively Blocked the Tumor Progression of Glioblastoma via Suppression of ERK/NF- $\kappa$ B Signaling Pathway. Am J Chin Med 2019;47:913-31.

21. Chen Y, Wang D, Peng H, et al. Epigenetically upregulated oncoprotein PLCE1 drives esophageal carcinoma angiogenesis and proliferation via activating the PI-PLC $\varepsilon-N F-\kappa B$ signaling pathway and VEGF-C/ Bcl-2 expression. Mol Cancer 2019;18:1. 